\title{
Demographic Trends in Korean Native Cattle Explained Using Bovine SNP50 Beadchip
}

\author{
Aditi Sharma*, Dajeong Lim, Han-Ha Chai, Bong-Hwan Choi, Yongmin Cho** \\ Division of Animal Genomics and Bioinformatics, National Institute of Animal Science, RDA, Wanju 55365, Korea
}

\begin{abstract}
Linkage disequilibrium (LD) is the non-random association between the loci and it could give us a preliminary insight into the genetic history of the population. In the present study LD patterns and effective population size ( $\mathrm{Ne}$ ) of three Korean cattle breeds along with Chinese, Japanese and Mongolian cattle were compared using the bovine Illumina SNP50 panel. The effective population size $(\mathrm{Ne})$ is the number of breeding individuals in a population and is particularly important as it determines the rate at which genetic variation is lost. The genotype data in our study comprised a total of 129 samples, varying from 4 to 39 samples. After quality control there were $\sim 29,000$ single nucleotide polymorphisms (SNPs) for which $r^{2}$ value was calculated. Average distance between SNP pairs was $1.14 \mathrm{Mb}$ across all breeds. Average $r^{2}$ between adjacent SNP pairs ranged between was 0.1 for Yanbian to 0.3 for Qinchuan. Effective population size of the breeds based on $r^{2}$ varied from 16 in Hainan to 226 in Yanbian. Amongst the Korean native breeds effective population size of Brindle Hanwoo was the least with $\mathrm{Ne}=59$ and Brown Hanwoo was the highest with $\mathrm{Ne}=83$. The effective population size of the Korean cattle breeds has been decreasing alarmingly over the past generations. We suggest appropriate measures to be taken to prevent these local breeds in their native tracts.
\end{abstract}

Keywords: cattle, Korea, linkage disequilibrium, SNP

\section{Introduction}

The availability of genome wide single nucleotide polymorphism (SNP) chips has made it possible to study the linkage disequilibrium (LD) patterns, effective population size ( $\mathrm{Ne})$, genome wide associations, etc. easily and effectively. Whole genome SNP panels are widely being used to study the genetic diversity in different livestock breeds [1-3]. LD and Ne form an important part in understanding the genetic diversity of any breed. Present day breeds are a result of adaptation and extensive selection for favorable traits. So, studying effective population size of breeds over several generations helps in understanding the genetic history and selection pressures that the population might have undergone in the history. Effective population size is particularly important in population and quantitative genetics and in evolutionary and conservation biology as it determines the rate at which genetic variation is lost [4].
Korean cattle are taurine type cattle available across the mainland and nearby islands. There are three main cattle breeds in Korea viz., Brown Hanwoo (BH), Brindle Hanwoo $(\mathrm{BNH})$, and Jeju Black (JB). BH is the main cattle breed of Korea and has an established breeding and selection program. The number of $\mathrm{BH}$ runs in millions whereas the other two breeds have few thousand animals only. A close relative of $\mathrm{BH}$ called Yanbian (YB) also exists close to the Korea and China border [5]. So, we included the YB breed in the study as it is the unselected breed and would provide a direct comparison between the selected Korean breeds and unselected breeds. Korean cattle are known to have migrated from North China and Mongolia and from Korea to Japan. Considering this fact we conducted this study to understand the LD patterns and the effective population size of the Korean breeds along with Chinese, Mongolian (MG), and Japanese breeds. It is already known that due to various reasons about $16 \%$ of the cattle breeds are already extinct and $30 \%$ face the risk of extinction. Understanding the LD 
and $\mathrm{Ne}$ in Korean cattle population will allow the researchers to make better strategies for conservation and breeding of these cattle.

\section{Methods}

\section{Ethics statement}

No ethics statement was required for the collection of DNA samples. DNA was extracted from blood samples obtained from different veterinary practitioners across the country with the permission of the owners. The blood samples were collected for routine veterinary procedures and not explicitly for the purpose of this study.

\section{Animals, genotyping, quality control, and LD and Ne estimation}

Blood samples were collected from $\mathrm{BH}, \mathrm{BNH}$, and JB. Twenty $(n=20)$ animals were sampled from each Korean breed. Genotype data of Korean cattle was merged with genotype data from six other Asian breeds that included $\mathrm{YB}$, Luxi (LX), Hainan (HN), Qinchuan (QC), Wagyu (WAG), and MG cattle. The data for these six Asian breeds was downloaded from dryad.org [6]. There were a total of 129 samples from the 9 Asian breeds in the study (Table 1).

Genomic DNA for genotyping assays was extracted from the blood sample using DNeasy 96 Blood and Tissue Kit (Qiagen, Valencia, CA, USA). DNA quantification was performed using a NanoDrop 1000 (Thermo Fisher Scientific Inc., Wilmington, DE, USA). DNA samples were submitted for genotyping with total DNA of $900 \mathrm{ng}, 260 / 280$ ratio $>1.8$, and DNA concentration of $20 \mathrm{ng} / \mu \mathrm{L}$. The genotyping for animals was done by the Animal Genome \& Bioinformatics Division of the National Institute of Animal Science, Rural Development Administration (RDA), Korea, using a BovineSNP50 BeadChip ver. 1 (Illumina, San Diego, CA, USA).

Table 1. Details of the cattle breeds used in the study

\begin{tabular}{lllrll}
\hline \multicolumn{1}{c}{ Breed } & Origin & $\begin{array}{l}\text { Breed } \\
\text { code }\end{array}$ & Samples & $\mathrm{r}^{2}$ & $\mathrm{Hs}$ \\
\hline Yanbian & China & YB & 39 & 0.054 & 0.441 \\
Luxi & China & LX & 5 & 0.245 & 0.297 \\
Hainan & China & HN & 4 & 0.304 & 0.163 \\
Qinchuan & China & QC & 4 & 0.311 & 0.318 \\
Wagyu & Japan & WAG & 12 & 0.199 & 0.338 \\
Brown Hanwoo & Korea & BH & 20 & 0.1 & 0.4 \\
Jeju Black & Korea & JB & 20 & 0.115 & 0.394 \\
Brindle Hanwoo & Korea & BNH & 20 & 0.126 & 0.385 \\
Mongolian & Mongolia & MG & 5 & 0.26 & 0.361 \\
\hline
\end{tabular}

$r^{2}$ is the average linkage disequilibrium across all distances and $\mathrm{Hs}$ is the heterozygosity. Hs was used as a measure of genetic diversity.
Plink ver. 1.07 (http://pngu.mgh.harvard.edu/ purcell/ plink/) [7] was used for the quality control of the raw genotype data. Quality control was performed for minor allele frequency, missingness, Hardy-Weinberg equilibrium (HWE), and genotyping quality. Beagle program [8] was used for imputation of the missing genotypes. Five thousand sixty-seven markers were excluded based on HWE test ( $\mathrm{p} \leq$ 0.0001 ) and further 1,825 SNPs were excluded based on frequency test (minor allele frequency $<0.01$ ). Final dataset consisted of a total of 28,708 SNPs. A total of 129 samples were analyzed in the study. SNeP program [9] was used to calculate pairwise LD and Ne. LD based Ne was calculated according to Sved [10] as below,

$$
\mathrm{r}^{2}=1 /(4 c \mathrm{Ne}+1)
$$

where $r^{2}$ is the $\mathrm{LD}, c$ is the recombination rate and $\mathrm{Ne}$ is the effective population size.

Population structure was studied using Fst and principal component analysis (PCA) as implemented in adegent $\mathrm{R}$ package [11]. Genetic distances among the pair of populations were calculated using "WC84" method implemented in Hierfstat [12].

\section{Results and Discussion}

In the present study, we observed a clear separation of Korean cattle breeds from other breeds. Based on WC84 genetic distances, Korean breeds were found to be closer to YB and WAG cattle breeds (Supplementary Fig. 1). Among all the breeds in the study, heterozygosity (Hs) was highest in YB (0.44) and lowest in HN (0.16). Amongst the Korean cattle BH (0.40) had the highest heterozygosity compared to JB (0.39) and BNH (0.38). The difference in heterozygosity amongst the Korean breeds was very small which suggests the genetic closeness of these populations. The heterozygosity in our study was higher than that reported by Strucken et al. [13] which could be because of different SNPs used in the final dataset.

Population differentiation and structure were studied using Fst and PCA. Highest Fst values were seen for HN which is azebu cattle from China. The lowest Fst value was observed for YB followed by the Korean breeds $\mathrm{BH}, \mathrm{BNH}$, and JB (Supplementary Fig. 2). Low Fst values in Korean populations suggest lack of population differentiation. This also suggests that these breeds could be interbreeding freely in the past. In PCA, WAG formed a separate cluster while Korean cattle still formed a close cluster along with YB (Supplementary Fig. 3). Both Fst based analysis and PCA showed that Korean breeds have yet not differentiated enough from one another. 
LD patterns in the 9 Asian cattle breeds were compared as it could help us understand the forces that structured the present day populations and will also reflect the past evolutionary and demographic events. LD across the genome gives an insight into the population history, the breeding system and the pattern of geographic subdivision [14]. In our study, the average distance between SNP pairs was $1.14 \mathrm{Mb}$ across all breeds. The LD decreased with increasing physical distance between the markers (Fig. 1). Estimates of LD based on average $r^{2}$ values varied from 0.1 for $Y B$ to 0.31 for QC (Table 1). Lowest level of LD in YB could be because it didn't undergo any artificial selection. Other cattle breeds in the study undergo moderate to intensive selection regimes. Among the Korean breeds, $\mathrm{BH}(0.1)$ had the lowest value of $\mathrm{LD}$ while $\mathrm{BNH}$ had the highest $\mathrm{LD}$ value of 0.13 . BH is the only Korean breed that has a dedicated breeding and selection program which could be the reason for low LD in this breed. Twenty BH Korea proven bulls (called KPN) are used throughout the country for artificial insemination of cows, thus leading to low LD. Similar pattern was observed by Edea et al. [15] in Korean cattle. Average LD in Japanese cattle, WAG, for all SNP pairs was found to be 0.31 which was less than the Chinese (with an exception of YB) and MG cattle but more than the Korean cattle breeds. WAG, which is a premium beef breed of Japan, has been maintained as a closed breed since $\sim 100$ years which could be one of the reasons for higher LD. LX, HN, QC and also MG breeds had higher LD than the other breeds which indicate their long

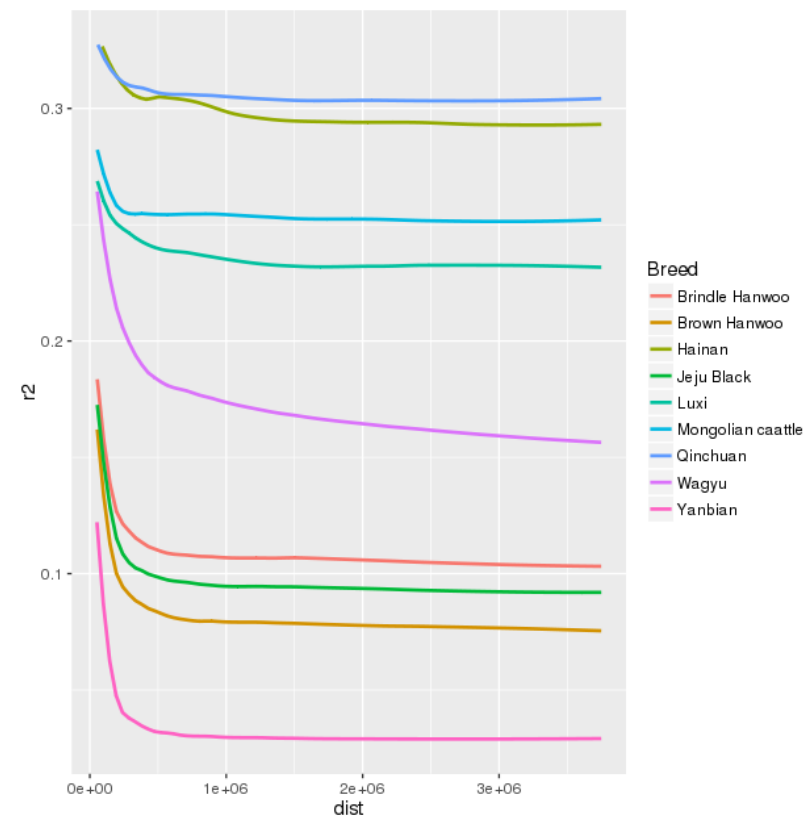

Fig. 1. Linkage disequilibrium plotted as a correlation between $r^{2}$ and genetic distance between single nucleotide polymorphisms in the 9 cattle breeds used in this study. history of domestication. The improvement of MG cattle began in 1949 by crossbreeding with shorthorn cattle (http://www.thecattlesite.com/breeds/beef/28/mongolian/). In contrast to Chinese and MG cattle, a dedicated breeding program for Korean cattle was started only in 1979 [16].

Effective population size is strongly associated with genetic variability and adaptation. So to better understand the size and structure of these cattle populations, effective population size was calculated using the LD estimates $\left(\mathrm{r}^{2}\right)$. All the cattle populations showed a declining trend in their effective population size (Fig. 2). Amongst all the breeds under study YB had the highest $\mathrm{Ne}=226$ until 13 generations ago while Chinese $\mathrm{HN}$ and QC had the lowest $\mathrm{Ne}$ of 16. According to a Food and Agriculture Organization of the United Nations (FAO) report (ftp://ftp.fao.org/docrep/fao/ 012/v6950t/v6950t02.pdf), HN cattle of china which is of indicine origin is categorized as an endangered breed and the total number of animals in the Hainan island has dropped considerably owing to economic reasons and lack of an organized effort. HN and QC were followed by MG, LX, and WAG in which Ne was 20, 23 and 37, respectively. Among the Korean cattle $\mathrm{BNH}$ had the smallest $\mathrm{Ne}=59$ until 13 generations ago and JB and $\mathrm{BH}$ had a $\mathrm{Ne}$ of 67 and 83 respectively. This is however considerably less than that reported by Lee et al. [17] and Li and Kim [18]. Lee et al. [17] reported $\mathrm{Ne}$ in $\mathrm{BH}$ to be 352 until 10 generations ago whereas Li and Kim [18] reported Ne to be 630 around 11 generations ago. Dang et al. [19] estimated Ne to be 47, 5 years ago from 2011. The trend of LD and Ne is the same as reported by $\mathrm{Li}$ and $\mathrm{Kim}$ [18] and Lee et al. [17] however the difference in estimated number could be attributed to

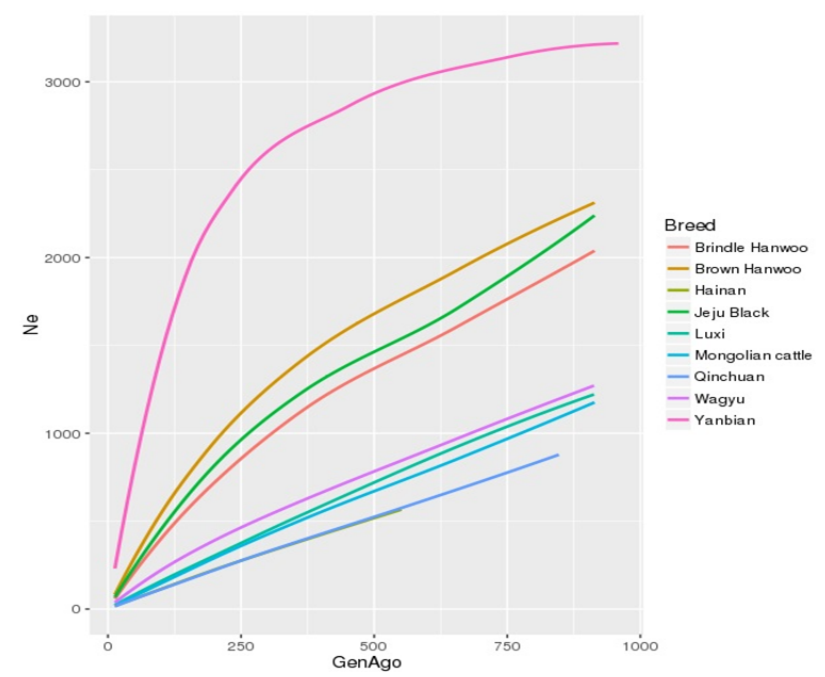

Fig. 2. Effective population size $(\mathrm{Ne})$ in the 9 Asian breeds. A decreasing trend in effective population size was observed. GenAgo, generations ago. 
number of samples used in the study and SNP quality control measures. The results might also differ because of the model used to study LD and Ne. In our study sample size was the limiting factor. Increase in sample size will further validate the results of the study.

The pattern of $\mathrm{LD}$ and $\mathrm{Ne}$ is considerably affected by the past historic events of the population. In this study we observed a sharp decline in the effective population size of all the cattle breeds. We observed a consistent decline in the size of cattle populations throughout all the generations however a sharp plunge in effective population size was observed at 50-100 generations ago. This was the time of formation of the current breeds. Further decline in $\mathrm{Ne}$ was observed at $\sim 50$ generations ago in Korean cattle. This was the time when selection and development of breeding programs had just begun. The modern day cattle genetic structure, $\mathrm{LD}$ and $\mathrm{Ne}$ is a result of the various historic events and extensive artificial and natural selection.

In our study we found a steep decline in the effective population size of cattle populations. Effective population size has been decreasing at an alarmingly high rate in Korean cattle population especially $\mathrm{BNH}$ and JB. We suggest strict actions and strategies to be adopted to increase the effective population size while maintaining the genetic diversity of the breeds.

\section{Supplementary materials}

Supplementary data including three figures can be found with this article online at http://www.genominfo.org/src/ sm/gni-14-230-s001.pdf.

\section{Acknowledgments}

Authors acknowledge the funds from 2016 Postdoctoral Fellowship Program of National Institute of Animal Science and "Cooperative Research Program for Agriculture Science \& Technology Development (Project No. PJ01022002)" of Rural Development Administration, Republic of Korea. Funding bodies had no role in the design of the study, collection, analysis, interpretation of data and in writing the manuscript.

\section{References}

1. Ben Jemaa S, Boussaha M, Ben Mehdi M, Lee JH, Lee SH. Genome-wide insights into population structure and genetic history of Tunisian local cattle using the Illumina bovinesnp50 beadchip. BMC Genomics 2015;16:677.

2. Aslam ML, Bastiaansen JW, Elferink MG, Megens HJ, Crooijmans RP, Blomberg LA, et al. Whole genome SNP discovery and analysis of genetic diversity in Turkey (Meleagris gallopavo). BMC Genomics 2012;13:391.
3. McKay SD, Schnabel RD, Murdoch BM, Matukumalli LK, Aerts J, Coppieters W, et al. An assessment of population structure in eight breeds of cattle using a whole genome SNP panel. BMC Genet 2008;9:37.

4. Saura M, Tenesa A, Woolliams JA, Fernández A, Villanueva B. Evaluation of the linkage-disequilibrium method for the estimation of effective population size when generations overlap: an empirical case. BMC Genomics 2015;16:922.

5. Choi JW, Choi BH, Lee SH, Lee SS, Kim HC, Yu D, et al. Whole-genome resequencing analysis of Hanwoo and Yanbian cattle to identify genome-wide SNPs and signatures of selection. Mol Cells 2015;38:466-473.

6. Decker JE, McKay SD, Rolf MM, Kim J, Molina Alcalá A, Sonstegard TS, et al. Worldwide patterns of ancestry, divergence, and admixture in domesticated cattle. PLoS Genet 2014;10:e1004254.

7. Purcell S, Neale B, Todd-Brown K, Thomas L, Ferreira MA, Bender D, et al. PLINK: a tool set for whole-genome association and population-based linkage analyses. Am J Hum Genet 2007;81:559-575.

8. Browning BL, Browning SR. A unified approach to genotype imputation and haplotype-phase inference for large data sets of trios and unrelated individuals. Am J Hum Genet 2009; 84:210-223.

9. Barbato M, Orozco-terWengel P, Tapio M, Bruford MW. SNeP: a tool to estimate trends in recent effective population size trajectories using genome-wide SNP data. Front Genet 2015;6:109.

10. Sved JA. Linkage disequilibrium and homozygosity of chromosome segments in finite populations. Theor Popul Biol 1971;2:125-141.

11. Jombart T. Adegenet: a R package for the multivariate analysis of genetic markers. Bioinformatics 2008;24:1403-1405.

12. Goudet J. HIERFSTAT, a package for R to compute and test hierarchical F-statistics. Mol Ecol Resour 2005;5:184-186.

13. Strucken EM, Lee SH, Jang GW, Porto-Neto LR, Gondro C. Towards breed formation by island model divergence in Korean cattle. BMC Evol Biol 2015;15:284.

14. Slatkin M. Linkage disequilibrium: understanding the evolutionary past and mapping the medical future. Nat Rev Genet 2008;9:477-485.

15. Edea Z, Dadi H, Kim SW, Dessie T, Lee T, Kim H, et al. Genetic diversity, population structure and relationships in indigenous cattle populations of Ethiopia and Korean Hanwoo breeds using SNP markers. Front Genet 2013;4:35.

16. Lee SH, Park BH, Sharma A, Dang CG, Lee SS, Choi TJ, et al. Hanwoo cattle: origin, domestication, breeding strategies and genomic selection. J Anim Sci Technol 2014;56:2.

17. Lee SH, Cho YM, Lim D, Kim HC, Choi BH, Park HS, et al. Linkage disequilibrium and effective population size in Hanwoo Korean cattle. Asian-Australas J Anim Sci 2011; 24:1660-1665.

18. Li Y, Kim JJ. Effective population size and signatures of selection using bovine 50K SNP chips in Korean native cattle (Hanwoo). Evol Bioinform Online 2015;11:143-153.

19. Dang CG, Lee JJ, Kim NS. Estimation of inbreeding coefficients and effective population size in breeding bulls of Hanwoo (Korean cattle). J Anim Sci Technol 2011;53:297-302. 